\title{
ANALYSIS OF WAVE INTERACTION WITH SUBMERGED ADJACENT PORO-ELASTIC BREAKWATERS
}

\author{
Yuan-Jyh Lan ${ }^{1}$, Tai-Wen Hsu ${ }^{1}$ and Ching-Yu Chen ${ }^{1}$
}

\begin{abstract}
In this study, the problem of wave interaction with two closely submerged adjacent porous elastic breakwaters is investigated theoretically. The porous elastic breakwaters are assumed to be homogeneous, isotropic and elastic. Lan and Lee's (2010) analytical solution is extended to the problem subject to proper boundary conditions. Using general solutions for each region and the matching boundary conditions, a set of simultaneous equations is thus developed and solved numerically. The present analytic solutions compare favorably with simplified cases of the poro-elastic submerged breakwater. Changes of the width of two adjacent breakwaters, the permeable coefficient effect on wave profile, and the effect of materials and configurations of breakwaters on wave variation are the focus of the present paper. The results show that a large amount of energy dissipates when the adjacent structures both have the same soft material and dimension of width with higher permeability. Different materials and shapes of structures could significantly influence the reflection coefficient.
\end{abstract}

Keywords: waves; submerged poro-elastic structures; closely adjacent breakwaters; interaction

\section{INTRODUCTION}

The increase of ecological and near-natural coastal engineering escalates requirements on structures and construction methods, and no longer constitutes the simple purpose of protection. Not only do the performing methods need to accommodate nature, but also various structural materials should be used from local environments. It is difficult to achieve both minimal impact on the environment and enough strength for practical protection. Most structures are usually constructed by rigid materials and attached to outer layers using ecological engineering methods in order to make them more similar to the natural environment. The outer layers of structures are usually composed of permeable and flexible material. This kind of structure can be regarded as composite structures, consisting of two or more different interaction mechanisms with wave motion. Due to the flexibility of soft materials, the deformation induced by water waves disturbs the flow field in the vicinity of the structure when compared with their impermeable reflective concrete counterparts (Lan et al., 2011). To understand wave transformation by two closely adjacent submerged breakwaters, the additional flexible effect of submerged adjacent permeable breakwaters on wave scattering and energy dissipation are investigated theoretically in this paper.

The study of wave interaction with poro-elastic structures is difficult because of the complexity of dynamic mechanisms in the poro-elastic medium and its surface. Relevant investigations have included flexible and permeable plates or breakwaters (Wang and Ren, 1993; Yip et al., 2002) and poro-elastic seabeds with infinite widths (Huang and Song, 1993; Chen et al., 1997; Tseng et al., 2008). The formulation of these studies has been simplified in that the particular structural size and unlimited width of seabed could maintain no change along the horizontal direction or thin poro-elastic plates would reduce the complexity of the flexible and permeable mechanisms. For the case of finite size breakwaters, Lan and Lee (2010) proposed an improved Biot's theory for evaluation of high permeable resistance introduced by Sollitt and Cross (1972). They analyzed the reflection, transmission and energy dissipation of monochromatic waves traveling over a single rectangular submerged poro-elastic structure. According to Lan and Lee (2010), Lan et al. (2011) obtained an analytical solution for wave scattering by a series of poro-elastic submerged structures, and accordingly, laboratory experiments were performed for this study.

In this paper, an analytical solution which is extended from Lan and Lee's theory was obtained for wave scattering by two closely adjacent poro-elastic submerged breakwaters. A limited approach is employed and the solution is in terms of orthogonal eigenfunctions. Influence parameters are analyzed to account for the reflection and transmission coefficients, while calculations from the analytical solution are compared with previous results. Key features of wave transformation affected by various adjacent breakwater widths, permeability coefficients, materials and configurations of breakwaters are also discussed.

\footnotetext{
${ }^{1}$ Dept. of Hydraulic and Ocean Engineering, National Cheng Kung University, 1 University Rd., Tainan, 701, TAIWAN (ROC)
} 


\section{PROBLEM FORMULATION}

Two rectangular shapes of submerged poro-elastic breakwaters with the same height $h$ are fixed closely adjacent on an impermeable seabed, and are subject to incident waves. Each of the poro-elastic breakwaters is assumed to be homogeneous, saturated and hydraulically isotropic. The schematic diagram is illustrated in Figure 1, where $d$ is the water depth, and $b_{I I}$ (upstream side) and $b_{I V}$ (downstream side) are structural widths, respectively. A two-dimensional Cartesian coordinate system is used with the original point located at the interface between the impermeable seabed surface and the center of the upstream breakwater, while the $x$-axis is pointed to the right, and the $z$-axis is pointed upward. Incident waves propagate in the negative $x$ direction. The wave profile $\eta_{I}$ and velocity potential function $\Phi_{I}$ can be expressed, respectively, as

$$
\begin{gathered}
\eta_{I}=\operatorname{Re}\left\{A_{I} e^{-i k_{0}\left(x-\frac{b_{I I}}{2}\right)} e^{-i \omega t}\right\} \\
\Phi_{I}=-i \frac{g A_{I}}{\omega} \frac{\cosh k_{0} z}{\cosh k_{0} d} e^{-i k_{0}\left(x-\frac{b_{I I}}{2}\right)} e^{-i \omega} \quad t
\end{gathered}
$$

where $\mathrm{Re}$ is the real part of a complex variable, $A_{I}$ the wave amplitude, $k_{0}$ the wavenumber, $\omega=2 \pi / T$ the angular frequency, $T$ the wave period, $t$ the time, $g$ gravitational acceleration, and $i=\sqrt{-1}$ is the complex unit.

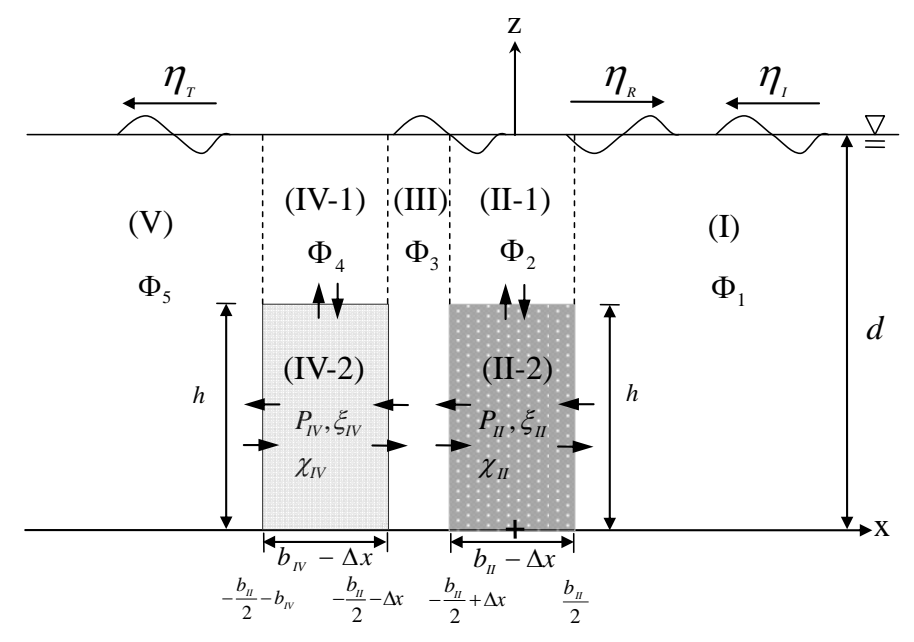

Figure 1. Sketch of the boundary value problem on waves passing submerged adjacent poro-elastic breakwaters.

Unlike composite breakwaters, submerged poro-elastic breakwaters are placed very in close proximity to each other; consequently, it is difficult to analyze the complicated physical properties of the two closely adjacent boundaries. Two assumptions are made on the two closely positioned breakwaters in order to derive analytical solutions. The assumptions are as follows: (1) A thin fluid layer with a tiny width of $2 \Delta x$ exists in two closely adjacent breakwaters; and (2) Movements of the two poro-elastic breakwaters do not interfere with each other.

To solve this problem, the study domain is divided into five fluid regions and two breakwater regions as shown in Figure 1, in which region (III) is the thin fluid domain between the two closely adjacent poro-elastic breakwaters. As $\Delta x$ approaches zero, the breakwaters shown in Figure 1 could tend to the submerged adjacent poro-elastic conditions.

\section{Governing equations for poro-elastic breakwaters}

The poro-elastic submerged breakwaters at regions (II)-2 and (IV)-2 are assumed to be homogeneous, isotropic and saturated with poro-elastic features. In this situation, the conservation of mass for the wave field is satisfied based on Verruijt's equation (1969): 


$$
\frac{\partial P}{\partial t}=-\frac{1}{n^{\prime} \beta}\left[\nabla \cdot\left(\frac{\partial \vec{d}^{*}}{\partial t}\right)+n^{\prime} \nabla \cdot \vec{Q}\right]
$$

where $P$ is the pore pressure, $n^{\prime}$ is the porosity of the poro-elastic medium, $\beta$ is the compressibility of the pore fluid, $\vec{Q}$ is the fluid velocity relative to the elastic solid, $\vec{d}^{*}=\xi \vec{i}+\chi \vec{j}$ is the elastic solid displacement, and $\xi$ and $\chi$ are components of displacement in the $x$ - and $z$-directions, respectively. Physically, equation (3) describes the change in pore pressure which is related to the dilation rates of the pore fluid and the elastic solid skeleton. The conservation of momentum follows Biot's theory (1956) written in the following form:

$$
\begin{gathered}
\nabla \cdot \tau^{*}-\left(1-n^{\prime}\right)(\nabla P)=\left(1-n^{\prime}\right) \rho_{s} \frac{\partial^{2} \vec{d}^{*}}{\partial t^{2}}-n^{\prime} \rho_{w} C_{a} \frac{\partial \vec{Q}}{\partial t}-n^{\prime} \omega \rho_{w} f \vec{Q} \\
-n^{\prime}(\nabla P)=n^{\prime} \rho_{w} \frac{\partial^{2} \vec{d}^{*}}{\partial t^{2}}+n^{\prime} \rho_{w} S \frac{\partial \vec{Q}}{\partial t}+n^{\prime} \omega \rho_{w} f \vec{Q}
\end{gathered}
$$

where $\nabla=(\partial / \partial x, \partial / \partial z)$ is the gradient operator, $f$ the linear friction factor, $\rho_{s}$ the density of elastic solid structure, $\rho_{w}$ the density of fluid, $S$ the virtual mass coefficient, and $\tau^{*}$ is the effective stress tensor of the poro-elastic media. It is noted that the linear friction factor $f$ is determined by the influence parameters of the kinematic viscosity of the fluid $v$, the intrinsic permeability $k_{p}$, the turbulent drag coefficient $C_{f}$ and the porosity $n^{\prime}$. In equations (4) and (5), Sollitt and Cross's theory (1972) and Lorentz's hypothesis have been applied to deal with the turbulent resistance force and linearize the governing equations, respectively. For further details, please refer to Lan and Lee (2010).

On the other hand, the relationship between the effective stresses and strains in the poro-elastic medium is governed by Hooke's law:

$$
\tau^{*}=\left\{\begin{array}{cc}
\sigma_{x}^{\prime} & \tau_{z x}^{\prime} \\
\tau_{x z}^{\prime} & \sigma_{z}^{\prime}
\end{array}\right\}=\left\{\begin{array}{cc}
2 G\left(\frac{\partial \xi}{\partial x}+\frac{\mu\left(\nabla \cdot \vec{d}^{*}\right)}{1-2 \mu}\right) & G\left(\frac{\partial \chi}{\partial x}+\frac{\partial \xi}{\partial z}\right) \\
G\left(\frac{\partial \chi}{\partial x}+\frac{\partial \xi}{\partial z}\right) & 2 G\left(\frac{\partial \chi}{\partial z}+\frac{\mu\left(\nabla \cdot \vec{d}^{*}\right)}{1-2 \mu}\right)
\end{array}\right\}
$$

where $\sigma_{x}^{\prime}, \sigma_{z}^{\prime}, \tau_{x z}^{\prime}$ and $\tau_{x z}^{\prime}$ are the effective stresses of the poro-elastic medium, respectively, $G=E /[2(1+\mu)]$ is the shear modulus, $\mu$ is Poisson's ratio, and $E$ is Young's modulus.

Equations (3)-(6) can be decoupled into three partial differential equations (PDEs) in $\xi$, $\chi$ and $P$ as follows (Lan et al. 2011):

$$
\begin{gathered}
\left\{\left[\nabla^{2}+\alpha_{1}{ }^{2}\right] \nabla^{2}+\alpha_{2}{ }^{4}\right\}\left(\nabla^{2}+\alpha_{3}{ }^{2}\right)\left\{\begin{array}{l}
\xi \\
\chi
\end{array}\right\}=0 \\
\left\{\left[\nabla^{2}+\alpha_{1}{ }^{2}\right] \nabla^{2}+\alpha_{2}{ }^{4}\right\} P=0
\end{gathered}
$$

The coefficients $\alpha_{1}, \alpha_{2}$ and $\alpha_{3}$ are, respectively, given by

$$
\begin{gathered}
\alpha_{1}^{2}=\omega^{2} \rho_{w} \beta(S+i f)+\frac{1-2 \mu}{2 G(1-\mu)}\left(\omega^{2}\right)\left\{\bar{\rho}+\rho_{w}\left[\frac{(S+i f)}{n^{\prime}}-2\right]\right\} \\
\alpha_{2}{ }^{4}=\frac{1-2 \mu}{2 G(1-\mu)}\left(\omega^{4} \rho_{w} \beta\right)\left[\bar{\rho}(S+i f)-n^{\prime} \rho_{w}\right]
\end{gathered}
$$




$$
\alpha_{3}{ }^{2}=\frac{\omega^{2}}{G}\left(\bar{\rho}-\frac{n^{\prime} \rho_{w}}{(S+i f)}\right)
$$

where $\bar{\rho}=\left(1-n^{\prime}\right) \rho_{s}+n^{\prime} \rho_{w}$ is the mean density of the poro-elastic medium. Therefore, the physical properties of the poro-elastic breakwaters in regions (II)-2 and (IV)-2 are denoted by the subscript 'II' and 'IV', respectively. We notice that equation (5) is used to obtain $\vec{Q}$ after $\xi, \chi$ and $P$ are solved.

\section{Governing equations for water waves}

The fluid domain is divided into regions (I), (II)-1, (III), (IV)-1, and (V). The velocity potentials satisfy the Laplace equation given by

$$
\nabla^{2} \Phi_{j}=0 \quad, j=1,2,3,4,5
$$

in which $\Phi_{1}=\Phi_{I}+\Phi_{R}$ is the velocity potential at region (I) and $\Phi_{R}$ is the reflected velocity potential. The velocity vector related to the potential function is determined by $\left(\vec{q}_{w}\right)_{j}=\Phi_{j}$.

\section{Boundary conditions}

The linearized free surface boundary condition reads as:

$$
\frac{\partial \Phi_{j}}{\partial z}-\frac{\omega^{2}}{g} \Phi_{j}=0, \text { on } z=d, j=1,2,3,4,5
$$

The bottom boundary conditions matches the non-slip condition:

$$
\left.\begin{array}{c}
\frac{\partial \Phi_{j}}{\partial z}=0, \quad z=0, \quad j=1,3,5 \\
n_{J}^{\prime} Q_{z, J}=0 \\
\xi_{J}=0 \\
\chi_{J}=0
\end{array}\right\}, z=0, J=I I, I V
$$

The boundary conditions on the breakwater surface are given by

$$
\begin{gathered}
\sigma_{z, J}^{\prime}=\tau_{x z, J}^{\prime}=0, z=h, J=I I, I V \\
\sigma_{x, I I}^{\prime}=\tau_{z x, I I}^{\prime}=0 \text {, on }(a) x=c_{1},(b) x=c_{2} \\
\sigma_{x, I V}^{\prime}=\tau_{z x, I V}^{\prime}=0, \text { on }(a) x=c_{3},(b) x=c_{4}
\end{gathered}
$$

where $c_{1}=b_{I I} / 2, c_{2}=-b_{I I} / 2+\Delta x, c_{3}=-b_{I I} / 2-\Delta x$ and $c_{4}=-b_{I I} / 2-b_{I V}$. The matching boundary conditions between any two closely adjoining regions are the continuity of both the pore pressure and normal flow flux, which are written as:

$$
\begin{aligned}
& \left.\begin{array}{c}
\Phi_{j}=\Phi_{j+1} \\
\frac{\partial \Phi_{j}}{\partial x}=\frac{\partial \Phi_{j+1}}{\partial x}
\end{array}\right\}, x=c_{j}, j=1,2,3,4 \\
& \left.\begin{array}{c}
P_{I I}=i \omega \quad \rho_{w} \Phi_{2 j-1} \\
n_{I I}^{\prime} Q_{x, I I}-i \omega \xi_{I I}=\frac{\partial \Phi_{2 j-1}}{\partial x}
\end{array}\right\}, x=c_{j}, \quad j=1,2
\end{aligned}
$$




$$
\begin{aligned}
& \left.\begin{array}{c}
P_{I V}=i \omega \quad \rho_{w} \Phi_{2 j+1} \\
n_{I V}^{\prime} Q_{x, I V}-i \omega \xi_{I V}=\frac{\partial \Phi_{2 j+1}}{\partial x}
\end{array}\right\}, x=c_{j+2}, j=1,2 \\
& \left.\begin{array}{cc}
P_{J}=i \omega & \rho_{w} \Phi_{j} \\
n_{J}^{\prime} Q_{z, J}-i \omega & \chi_{J}=\frac{\partial \Phi_{j}}{\partial z}
\end{array}\right\}, z=h, j=2,4, \quad J=I I, I V
\end{aligned}
$$

\section{Formulation of the homogeneous boundary value problem}

The pressure and flow flux continuous boundary conditions between regions (II)-1 and (II)-2, and regions (IV)-1 and (IV)-2 are non-homogeneous. Based on the linear assumption, the physical properties in regions (II)-1 and (II)-2 and regions (IV)-1 and (IV)-2 are divided into two parts, i.e., $\Phi_{2}=\Phi_{2}^{a}+\Phi_{2}^{b}, P_{I I}=P_{I I}^{a}+P_{I I}^{b}, \xi_{I I}=\xi_{I I}^{a}+\xi_{I I}^{b}, \chi_{I I}=\chi_{I I}^{a}+\chi_{I I}^{b}, Q_{x, I I}=Q_{x, I I}^{a}+Q_{x, I I}^{b}, Q_{z, I I}=Q_{z, I I}^{a}+Q_{z, I I}^{b}$, $\Phi_{4}=\Phi_{4}^{a}+\Phi_{4}^{b}, P_{I V}=P_{I V}^{a}+P_{I V}^{b}, \xi_{I V}=\xi_{I V}^{a}+\xi_{I V}^{b}, \chi_{I V}=\chi_{I V}^{a}+\chi_{I V}^{b}, Q_{x, I V}=Q_{x, I V}^{a}+Q_{x, I V}^{b}, Q_{z, I V}=Q_{z, I V}^{a}$ $+Q_{z, I V}^{b}$, and so on. Defining superscripts ' $a$ ' and ' $b$ ', the non-homogeneous boundary value problem can be modified by separating regions (II)-1 and (II)-2 to sub-regions (II)-1-a, (II)-1-b, (II)-2-a and (II)-2-b, respectively. Regions (II)-1-a and (II)-2-a satisfy the vertical homogeneous boundary conditions, while regions (II)-1-b and (II)-2-b fit the horizontal homogeneous boundary conditions. Following this procedure, the homogeneous boundary value problem of waves propagating over adjacent poro-elastic submerged structures is solved analytically. For further details refer to Lan and Lee (2010).

\section{METHOD OF SOLUTION}

To solve the boundary value problem mentioned above, we adopt calculus of the limit operator $\lim _{\Delta x \rightarrow 0}$ to whole derivations and solutions. The velocity potentials $\Phi_{R}, \Phi_{3}$ and $\Phi_{5}$ satisfying the Laplace equations along with the free surface and bottom conditions can be obtained formally by an eigenfunction expansion as follows:

$$
\begin{gathered}
\Phi_{R}=\sum_{n=0}^{\infty} D_{n(I)} e^{-\mu_{n}\left(x-\frac{b_{I I}}{2}\right)} \cos \mu_{n} z e^{-i \omega t} \\
\Phi_{3}=\sum_{n=0}^{\infty}\left[D_{n(I I I)}^{(1)} e^{\mu_{n}\left(x+\frac{b_{I I}}{2}\right)}+D_{n(I I I)}^{(2)} e^{-\mu_{n}\left(x+\frac{b_{I I}}{2}\right)}\right] \cos \mu_{n} z e^{-i \omega t} \\
\Phi_{5}=\sum_{n=0}^{\infty} D_{n(V)} e^{\mu_{n}\left(x+\frac{b_{I I}}{2}+b_{I V}\right)} \cos \mu_{n} z e^{-i \omega t}
\end{gathered}
$$

where $\mu_{0}=-i k_{0}$. The eigenvalues $\mu_{n}$ are determined by the dispersion relation as $\omega^{2}=$ $-g \mu_{n} \tan \mu_{n} d, n=0,1,2, \ldots$.

Note that both the reflected velocity potential $\Phi_{R}$ and the transmitted velocity potential $\Phi_{5}$ include propagating $(n=0)$ and evanescent $(n \geq 1)$ modes. Thus, the reflection and transmission coefficients can be evaluated by $K_{R}=\left|D_{0(I)}\right| \omega \cosh k_{0} d /\left(g A_{I}\right)$ and $K_{T}=\left|D_{0(V)}\right| \omega \cosh k_{0} d /\left(g A_{I}\right)$, respectively. Energy dissipation is estimated using wave energy conservation and given by $E_{f}=1-\left(K_{R}^{2}+K_{T}^{2}\right)$.

In a similar way, the expansions of $\Phi_{2}^{a}$ and $\Phi_{2}^{b}$ at region (II)-1 and $\Phi_{4}^{a}$ and $\Phi_{4}^{b}$ at region (IV)-1 are obtained and 


$$
\begin{gathered}
\Phi_{2 \ell}^{a}=\sum_{n=0}^{\infty}\left[A_{0 n(j-1)}^{a} e^{\mu_{n(j-1)}^{a}\left(x+(-1)^{\ell} \frac{b_{I I}}{2}\right)}+B_{0 n(j-1)}^{a} e^{-\mu_{n(j-1)}^{a}\left(x+(-1)^{\ell} \frac{b_{I I}}{2}\right)}\right] \\
\cos \mu_{n(j-1)}^{a}(z-h) e^{-i \omega t}, \quad j=I I, I V, \ell=1,2 \\
\Phi_{2 \ell}^{b}=\sum_{n=0}^{\infty} D_{0 n(j-1)}^{b}\left\{C_{0 n(j-1)}^{b}\left[\delta_{0 n}(z-d-1)+e^{\mu_{n(j-1)}^{b}(z-d)}\right]\right\} \\
\cos \mu_{n(j-1)}^{b}\left(x+(-1)^{\ell} \frac{b_{I I}}{2}\right) e^{-i \omega t}, \quad j=I I, I V, \quad \ell=1,2
\end{gathered}
$$

where $\mu_{n(I I-1)}^{b}=n \pi / b_{I I}, \mu_{n(I V-1)}^{b}=n \pi / b_{I V} \quad(n=0,1,2, \ldots), C_{00(I I-1)}^{b}=C_{00(I V-1)}^{b}=\omega^{2} / g$, and

$$
C_{00(j-1)}^{b}=\left(\mu_{n(j-1)}^{b}+\frac{\omega^{2}}{g}\right) /\left(\mu_{n(j-1)}^{b}-\frac{\omega^{2}}{g}\right), n=1,2,3, \ldots, \quad j=I I, I V
$$

in which $\delta_{0 n}$ is the Kronecker delta function. The eigenvalues $\mu_{n(I I-1)}^{a}$ and $\mu_{n(I V-1)}^{a}$ are determined by the dispersion relation

$$
\omega^{2}=-g \mu_{n(j-1)}^{a} \tan \mu_{n(j-1)}^{a}(d-h), n=0,1,2, \ldots, j=I I, I V
$$

The solutions of the system in regions (II)-2 and (IV)-2 are obtained by means of the method of separation of variables. The general solutions of the displacement components and pore pressure, satisfying the homogeneous matching boundary conditions, are given by

$$
\begin{aligned}
& \xi_{j}^{a}=\sum_{n=0}^{\infty}-\left\{\delta_{1 n(j-2)}^{a}\left(A_{1 n(j-2)}^{a} e^{\delta_{1 n(j-2)}^{a}\left(x+(-1)^{\frac{b}{2}} \frac{b_{j}}{2}\right)}-A_{2 n(j-2)}^{a} e^{-\delta_{1 n(j-2)}^{a}\left(x+(-1)^{\frac{b}{2}} \frac{b_{j}}{2}\right)}\right)\right. \\
& +\delta_{2 n(j-2)}^{a}\left(A_{3 n(j-2)}^{a} e^{\delta_{2 n(j-2)}^{a}\left(x+(-1)^{\ell} \frac{b_{j}}{2}\right)}-A_{4 n(j-2)}^{a} e^{-\delta_{2 n(j-2)}^{a}\left(x+(-1)^{\ell} \frac{b_{j}}{2}\right)}\right) \\
& \left.+\left(\frac{\hat{\mu}_{n(j-2)}^{a}{ }^{2}}{\delta_{3 n(j-2)}^{a}}\right)\left(A_{5 n(j-2)}^{a} e^{\delta_{3 n(j-2)}^{a}\left(x+(-1)^{\ell} \frac{b_{j}}{2}\right)}-A_{6 n(j-2)}^{a} e^{-\delta_{3 n(j-2)}^{a}\left(x+(-1)^{\ell} \frac{b_{j}}{2}\right)}\right)\right\} \\
& \cos \hat{\mu}_{n(j-2)}^{a}(z-h) e^{-i \omega t}, \quad j=I I, I V, \ell=1,2 \\
& \chi_{j}^{a}=\sum_{n=0}^{\infty} \hat{\mu}_{n(j-2)}^{a}\left\{\left(A_{1 n(j-2)}^{a} e^{\delta_{1 n(j-2)}^{a}\left(x+(-1)^{\ell} \frac{b_{j}}{2}\right)}+A_{2 n(j-2)}^{a} e^{-\delta_{1 n(j-2)}^{a}\left(x+(-1)^{\frac{b}{b}}\right.} \frac{b_{j}}{2}\right)\right) \\
& +\left(A_{3 n(j-2)}^{a} e^{\delta_{2 n(j-2)}^{a}\left(x+(-1)^{\ell} \frac{b_{j}}{2}\right)}+A_{4 n(j-2)}^{a} e^{-\delta_{2 n(j-2)}^{a}\left(x+(-1)^{\ell} \frac{b_{j}}{2}\right)}\right) \\
& \left.+\left(A_{5 n(j-2)}^{a} e^{\delta_{3 n(j-2)}^{a}\left(x+(-1)^{\ell} \frac{b_{j}}{2}\right)}+A_{6 n(j-2)}^{a} e^{-\delta_{3 n(j-2)}^{a}\left(x+(-1)^{\ell} \frac{b_{j}}{2}\right)}\right)\right\} \\
& \sin \hat{\mu}_{n(j-2)}^{a}(z-h) e^{-i \omega t}, \quad j=I I, I V, \ell=1,2
\end{aligned}
$$




$$
\begin{aligned}
P_{j}^{a}= & \sum_{n=0}^{\infty} \omega^{2} \rho_{w}\left(\frac{S_{j}+i f_{j}}{n^{\prime}}-1\right) \\
& \left\{\Lambda_{1 n(j-2)}^{a}\left(A_{1 n(j-2)}^{a} e^{\delta_{1 n(j-2)}^{a}\left(x+(-1)^{\prime} \frac{b_{j}}{2}\right)}+A_{2 n(j-2)}^{a} e^{-\delta_{1 n(j-2)}^{a}\left(x+(-1)^{\frac{b^{\prime}}{2}} \frac{b_{j}}{2}\right)}\right)\right. \\
& \left.+\Lambda_{2 n(j-2)}^{a}\left(A_{3 n(j-2)}^{a} e^{\delta_{2 n(j-2)}^{a}\left(x+(-1)^{\prime} \frac{b_{j}}{2}\right)}+A_{4 n(j-2)}^{a} e^{-\delta_{2 n(j-2)}^{a}\left(x+(-1)^{\frac{b_{j}}{2}}\right)}\right)\right\} \\
& \cos \hat{\mu}_{n(j-2)}^{a}(z-h) e^{-i \omega t}, \quad j=I I, I V, \quad \ell=1,2
\end{aligned}
$$

where

$$
\hat{\mu}_{n(j-2)}^{a}=n \pi / h, \quad n=0,1,2, \ldots, \quad j=I I, I V
$$

Similarly

$$
\begin{aligned}
& \xi_{j}^{b}=\sum_{n=0}^{\infty} \hat{\mu}_{n(j-2)}^{b}\left\{\left(A_{1 n(j-2)}^{b} e^{\delta_{\ln (j-2)}^{b}(z-h)}+A_{2 n(j-2)}^{b} e^{-\delta_{\ln (j-2)}^{b}(z-h)}\right)\right. \\
& +\left(A_{3 n(j-2)}^{b} e^{\delta_{2 n(j-2)}^{b}(\mathrm{z}-h)}+A_{4 n(j-2)}^{b} e^{-\delta_{2 n(j-2)}^{b}(\mathrm{z}-h)}\right) \\
& \left.+\left(A_{5 n(j-2)}^{b} e^{\delta_{3 n(j-2)}^{b}(\mathrm{z}-h)}+A_{6 n(j-2)}^{b} e^{-\delta_{3 n(j-2)}^{b}(\mathrm{z}-h)}\right)\right\} \\
& \sin \hat{\mu}_{n(j-2)}^{b}\left(x+(-1)^{\ell} \frac{b_{j}}{2}\right) e^{-i \omega t}, \quad j=I I, I V, \ell=1,2 \\
& \chi_{j}^{b}=\sum_{n=0}^{\infty}-\left\{\delta_{1 n(j-2)}^{b}\left(A_{1 n(j-2)}^{b} e^{\delta_{1 \ln (j-2)}^{b}(\mathrm{z}-h)}-A_{2 n(j-2)}^{b} e^{-\delta_{\ln (j-2)}^{b}(\mathrm{z}-h)}\right)\right. \\
& +\delta_{2 n(j-2)}^{b}\left(A_{3 n(j-2)}^{b} e^{\delta_{2 n(j-2)}^{b}(\mathrm{z}-h)}-A_{4 n(j-2)}^{b} e^{-\delta_{2 n(j-2)}^{b}(\mathrm{z}-h)}\right) \\
& \left.+\left(\frac{\hat{\mu}_{n(j-2)}^{b}}{\delta_{3 n(j-2)}^{b}}\right)\left(A_{1 n(j-2)}^{b} e^{\delta_{1 n(j-2)}^{b}(\mathrm{z}-h)}-A_{2 n(j-2)}^{b} e^{-\delta_{1 n(j-2)}^{b}(\mathrm{z}-h)}\right)\right\} \\
& \cos \hat{\mu}_{n(j-2)}^{b}\left(x+(-1)^{\ell} \frac{b_{j}}{2}\right) e^{-i \omega t}, \quad j=I I, I V, \ell=1,2 \\
& P_{j}^{b}=\sum_{n=0}^{\infty} \omega^{2} \rho_{w}\left(\frac{S_{j}+i f_{j}}{n_{j}^{\prime}}-1\right) \\
& \left\{\Lambda_{1 n(j-2)}^{b}\left(A_{1 n(j-2)}^{b} e^{\delta_{1 n(j-2)}^{b}(z-h)}+A_{2 n(j-2)}^{b} e^{-\delta_{1 n(j-2)}^{b}(z-h)}\right)\right. \\
& \left.+\left(A_{3 n(j-2)}^{b} e^{\delta_{2 n(j-2)}^{b}(\mathrm{z}-h)}+A_{4 n(j-2)}^{b} e^{-\delta_{2 n(j-2)}^{b}(\mathrm{z}-h)}\right)\right\} \\
& \cos \mu_{n(j-2)}^{b}\left(x+(-1)^{\ell} \frac{b_{j}}{2}\right) e^{-i \omega t}, \quad j=I I, I V, \ell=1,2
\end{aligned}
$$

where

$$
\hat{\mu}_{n(j-2)}^{b}=n \pi / b_{j}, \quad n=0,1,2, \ldots, \quad j=I I, I V
$$




$$
\begin{aligned}
& \left(\delta_{\ell n(j-2)}^{\psi}\right)^{2}=\left[\left(\hat{\mu}_{n(j-2)}^{\psi}\right)^{2}-\frac{\left(\alpha_{1}\right)_{j}^{2}}{2}\right]+(-1)^{\ell} \sqrt{\frac{\left(\alpha_{1}\right)_{j}^{4}}{4}-\left(\alpha_{2}\right)_{j}^{4}}, \\
& \psi=a, b, \quad \ell=1,2, \quad j=I I, I V \\
& \left(\delta_{3 n(j-2)}^{\psi}\right)^{2}=\left(\hat{\mu}_{n(j-2)}^{\psi}\right)^{2}-\left(\alpha_{3}\right)_{j}^{2}, \quad \psi=a, b, \quad j=I I, I V \\
& \Lambda_{\ell n(j-2)}^{\psi}=\frac{\left(\hat{\mu}_{n(j-2)}^{\psi}\right)^{2}-\left(\delta_{\ell n(j-2)}^{\psi}\right)^{2}}{\left(\hat{\mu}_{n(j-2)}^{\psi}\right)^{2}-\left(\delta_{\ell n(j-2)}^{\psi}\right)^{2}-\omega^{2} \rho_{w} \beta_{j}\left(S_{j}+i f_{j}\right)}, \\
& \psi=a, b, \quad \ell=1,2, \quad j=I I, I V
\end{aligned}
$$

The quantities $D_{n(I)}, D_{n(I I I)}^{(1)}, D_{n(I I I)}^{(2)}, D_{n(V)}, A_{0 n(j-1)}^{a}, B_{0 n(j-1)}^{a}, D_{0 n(j-1)}^{b}, A_{1 n(j-2)}^{a} \sim A_{6 n(j-2)}^{a}$ and $A_{1 n(j-2)}^{b} \sim A_{6 n(j-2)}^{b}(j=I I, I V)$ are 34 unknowns to be determined by approximations of power series. It is noted that equations (23) (27), (30) (32) and (34) (36) form a linear system of 34 equations. These unknowns can be obtained using the remaining matching boundary conditions together with the orthogonal eigenvalues $\mu_{n}, \mu_{n(j-1)}^{a}, \mu_{n(j-1)}^{b}, \hat{\mu}_{n(j-2)}^{a}$ and $\hat{\mu}_{n(j-2)}^{b}(j=I I, I V)$.

\section{VERIFICATION}

Even though the two closely adjacent poro-elastic submerged breakwaters are placed near a composite breakwater, it is plausible to consider the adjacent breakwaters as a single structural system in near rigid and homogeneous conditions. To compare the accuracy of the analytical solution with previous studies, the adjacent breakwaters are set at the same widths, i.e. $b_{I I}=b_{I V}=b / 2, \quad b$ is total structural width, and the material properties, i.e. $n_{I I}^{\prime}=n_{I V}^{\prime}=n^{\prime}, \beta_{I I}=\beta_{I V}=\beta, C_{f(I I)}=C_{f(I V)}=C_{f}$, $C_{a(I I)}=C_{a(I V)}=C_{a}, G_{I I}=G_{I V}=G, k_{p(I I)}=k_{p(I V)}=k_{p}, \mu_{I I}=\mu_{I V}=\mu$ and $\rho_{s(I I)}=\rho_{s(I V)}=\rho_{s}$.

Two cases with shear modulus $G=5 \times 10^{4} \mathrm{~N} / \mathrm{m}^{2}$ and $G=1 \times 10^{4} \mathrm{~N} / \mathrm{m}^{2}$ are calculated, in which $A_{I} / d=0.05, b / d=2, h / d=0.5, n^{\prime}=0.4, C_{f}=0.2, C_{a}=0.015, k_{p}=2.28 \times 10^{-6} \mathrm{~m}^{2}, \mu=0.333$, $\rho_{\mathrm{s}}=2650 \mathrm{~kg} / \mathrm{m}^{3}, \rho_{\mathrm{w}}=1000 \mathrm{~kg} / \mathrm{m}^{3}, v=1.12 \times 10^{-6} \mathrm{~m}^{2} / \mathrm{s}$, and $\beta=4.35 \times 10^{-10} \mathrm{~m}^{2} / \mathrm{N}$. The results of wave reflection, $K_{R}$, and transmission, $K_{T}$, versus $k_{0} d$ are presented in Figures 2 and 3 . The analytic solution obtained by Lan and Lee (2010) is shown in the same figure for comparison. Figure 2 shows the case of a harder poro-elastic breakwater $\left(G=5 \times 10^{4} \mathrm{~N} / \mathrm{m}^{2}\right)$ and the results of the present analytic solution agree fairly well. For the smaller shear modulus ( $G=1 \times 10^{4} \mathrm{~N} / \mathrm{m}^{2}$ ), shown in Figure 3 , in which the effect of the interface elasticity of two closely adjacent breakwaters on wave transformation is obvious, the transmission coefficient $K_{T}$ decreases, while the reflection coefficient $K_{R}$ increases as the number of reflected oscillation patterns increases. This implies that the phase lag of motions of the two poro-elastic breakwaters result in different wave transformations.

\section{RESULTS AND COMPARISONS}

In this section, the effects of the two closely adjacent poro-elastic submerged breakwaters on wave transformation including wave reflection, transmission and energy dissipation are intensively studied. This study focused on the investigation of changes of width of two adjacent breakwaters, the permeability of materials, and the shapes of breakwaters on wave transformation. The input conditions of poro-elastic structures and water waves used for the computations are: $k_{0} d=1.5, A_{I} / d=0.05$, $b / d=2, h / d=0.5, \rho_{w}=1000 \mathrm{~kg} / \mathrm{m}^{3}, v=1.12 \times 10^{-6} \mathrm{~m}^{2} / \mathrm{s}, \quad \beta_{1}=\beta_{2}=4.35 \times 10^{-10} \mathrm{~m}^{2} / \mathrm{N}$, $\rho_{s(I I)}=\rho_{s(I V)}=2650 \mathrm{~kg} / \mathrm{m}^{3}, n_{I I}^{\prime}=n_{I V}^{\prime}=0.4, \mu_{I I}=\mu_{I V}=0.333, C_{f(I I)}=C_{f(I V)}=0.2$, and $C_{a(I I)}=C_{a(I V)}=0.015$. It is noted that the shear modulus $G=1 \times 10^{4} \mathrm{~N} / \mathrm{m}^{2}$ is selected to represent elastic structures, while the condition of $G=5 \times 10^{5} \mathrm{~N} / \mathrm{m}^{2}$ approaches rigid materials. 


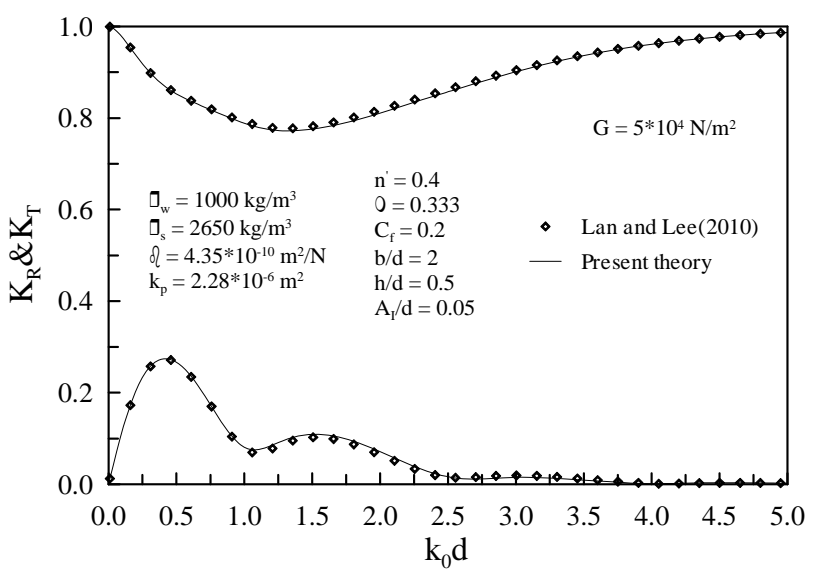

Figure 2. Comparisons between the present theory and solutions of Lan and Lee (2010) $\left(G=5 \times 10^{4} \mathrm{~N} / \mathrm{m}^{2}\right)$.

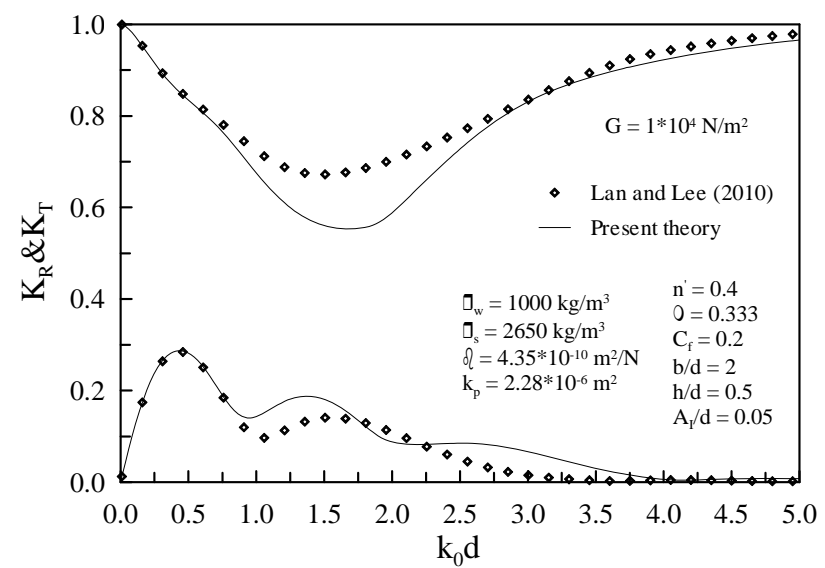

Figure 3. Comparisons between the present theory and solutions of Lan and Lee (2010) $\left(G=1 \times 10^{4} \mathrm{~N} / \mathrm{m}^{2}\right)$.

(a) Effect of breakwater widths with the same poro-elastic materials

To investigate the influence of width of two closely adjacent breakwaters with the same poroelastic material ( $G_{I I}=G_{I V}=1 \times 10^{4} \mathrm{~N} / \mathrm{m}^{2}$ ) on wave transformation, the total structural width is fixed as $b / d=2$, while three combinations of two closely adjacent breakwater widths, (1) $b_{I I} / d=0.5$, $b_{I V} / d=1.5$, (2) $b_{I I} / d=1, b_{I V} / d=1$, and (3) $b_{I I} / d=1.5, b_{I V} / d=0.5$, are computed in the study. Note that the first combination stands for the interface of two closely adjacent breakwaters closer to upstream, the second one is placed at the middle of the breakwater system, and the last one is positioned closer to downstream. Figure 4 shows the effect of two closely adjacent breakwater widths on $K_{R}, K_{T}$ and energy dissipation $E_{f}$ for higher material permeability $k_{p}=2.28 \times 10^{-6} \mathrm{~m}^{2}$. As the two closely adjacent breakwater width changes, the oscillation patterns of the wave reflection coefficient varies significantly within the range of $0.6<k_{0} d<4.0$. The lowest wave transmission coefficient $K_{T}$ and the largest energy dissipation $E_{f}$ respectively occur in $1.2<k_{0} d<2.6$ and $1.4<k_{0} d<2.4$ for the second combination of $b_{I I} / d=1$ and $b_{I V} / d=1$, respectively. For the first and third combination of two closely adjacent breakwater widths, the variation of $K_{T}$ and $E_{f}$ are quite similar. 

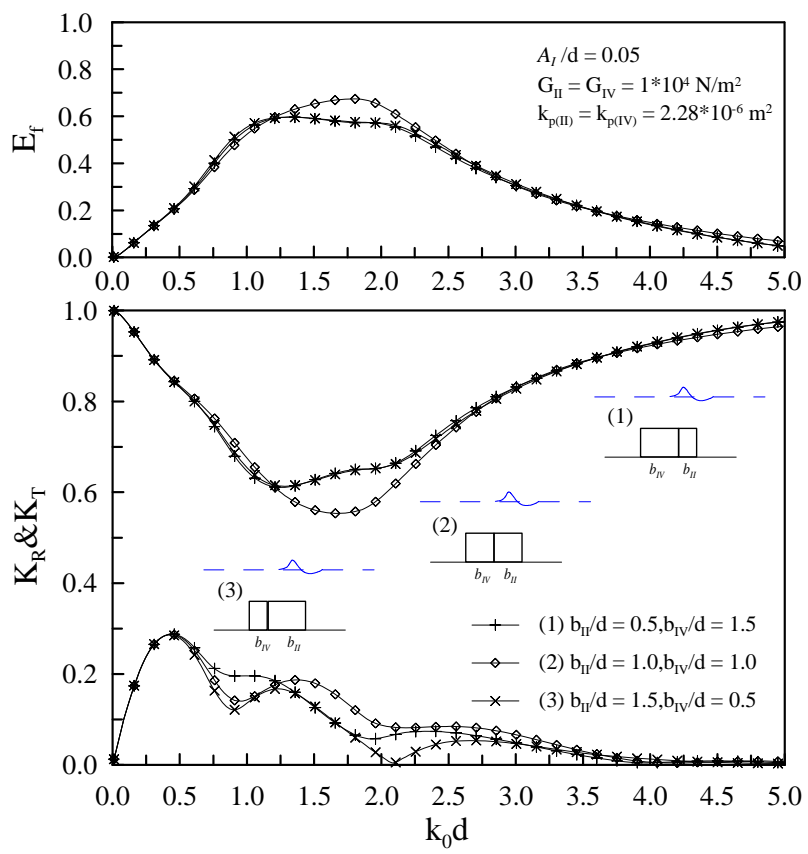

Figure 4. Effect of adjacent breakwater widths with the same poro-elastic material on $K_{R}$, $K_{T}$, and $E_{f}$ $\left(k_{p(I I)}=k_{p(I V)}=2.28 \times 10^{-6} \mathrm{~m}^{2}\right)$.

\section{(b) Effect of breakwater widths with different poro-elastic materials}

To examine the influence of different poro-elastic structures on wave reflection $K_{R}$, transmission $K_{T}$ and energy dissipation $E_{f}$, an elastic material $\left(G=1 \times 10^{4} \mathrm{~N} / \mathrm{m}^{2}\right.$ ) and a quasi-rigid one ( $G=5 \times 10^{5} \mathrm{~N} / \mathrm{m}^{2}$ ) with the same permeability are used in the calculation. The breakwater system having the width $b / d=2$ includes the rigid and elastic breakwaters by three combinations of two closely adjacent breakwater widths: (1) $b_{I I} / d=0.5, b_{I V} / d=1.5$, (2) $b_{I I} / d=1, b_{I V} / d=1$ and (3) $b_{I I} / d=1.5, b_{I V} / d=0.5$, in which the subscript 'II' denotes the upstream breakwater. The variation in $K_{R}, K_{T}$ and $E_{f}$ against $k_{0} d$ for various combinations of two closely adjacent breakwater widths with the same structural width $b$ are demonstrated in Figures 5 8. Notably Figures 5 and 6 show the results for the rigid structure located upstream, while Figures 7 and 8 depict the downstream cases.

For the case of a higher permeability ( $k_{p}=2.28 \times 10^{-6} \mathrm{~m}^{2}$ ), Figures 5 and 7 show that the shape of adjacent breakwater widths significantly affect the reflection coefficient $K_{R}$, while it rarely influences the transmission coefficient $K_{T}$ and energy dissipation $E_{f}$ regardless of whether the breakwater system locates rigid breakwater upstream or not. The increase of width of elastic breakwater produces broader bandwidths of the transmission coefficient and energy dissipation. The reflection coefficient oscillates obviously for the rigid breakwater placed upstream, but a low reflection coefficient occurs around the region near $k_{0} d=1.0$. In the case of the poro-elastic breakwater placed upstream, the oscillating phenomenon of the reflection coefficient is not significant. This is due to the fact that a higher permeability associated with elasticity could alter the reflected oscillation pattern. For a lower permeability ( $k_{p}=2.28 \cdot 10^{-9} \mathrm{~m}^{2}$ ), the results presented in Figures 6 and 8 show that the shape of two closely adjacent breakwater widths on wave reflection coefficient, wave transmission coefficient and energy dissipation is not noticeable. Furthermore, a larger width of poro-elastic breakwater increases the elastic effect on wave transformation and results in smaller values of $K_{T}$ and higher $K_{R}$ and $E_{f}$ in certain regions. Elastic structural width decreases could lead to peak values of $K_{T}$ and $E_{f}$ for a 
higher $k_{0} d$. The reflection coefficient oscillates more obviously when an increase in the elastic structural width with a lower permeability occurs.
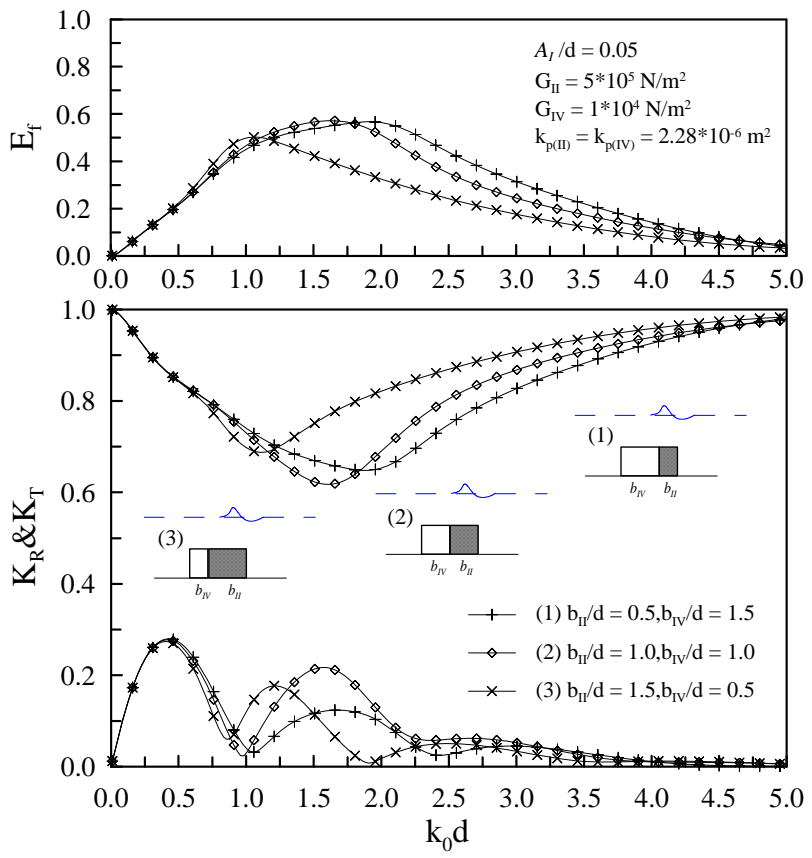

Figure 5. Effect of adjacent breakwater widths with different poro-elastic materials on $K_{R}, K_{T}, E_{f}$ $\left(G_{I I}=5 \times 10^{5} \mathrm{~N} / \mathrm{m}^{2}, G_{I V}=1 \times 10^{4} \mathrm{~N} / \mathrm{m}^{2}\right.$, and $\left.k_{p(I I)}=k_{p(I V)}=2.28 \times 10^{-6} \mathrm{~m}^{2}\right)$.
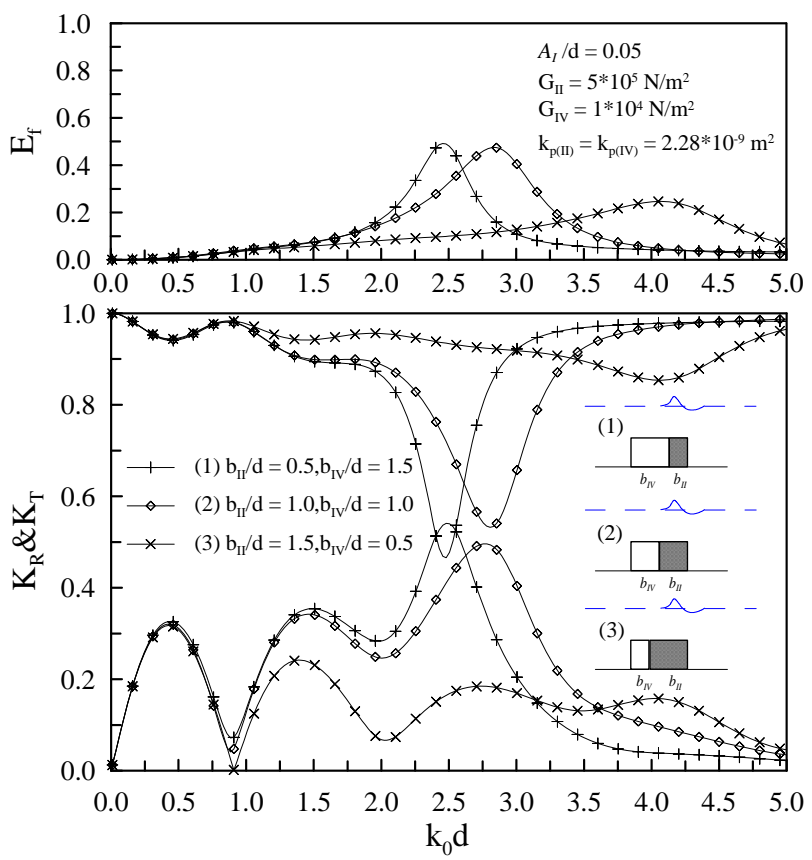

Figure 6. Effect of adjacent breakwater widths with different poro-elastic materials on $K_{R}, K_{T}, E_{f}$ $\left(G_{I I}=5 \times 10^{5} \mathrm{~N} / \mathrm{m}^{2}, G_{I V}=1 \times 10^{4} \mathrm{~N} / \mathrm{m}^{2}\right.$, and $\left.k_{p(I I)}=k_{p(I V)}=2.28 \times 10^{-9} \mathrm{~m}^{2}\right)$. 

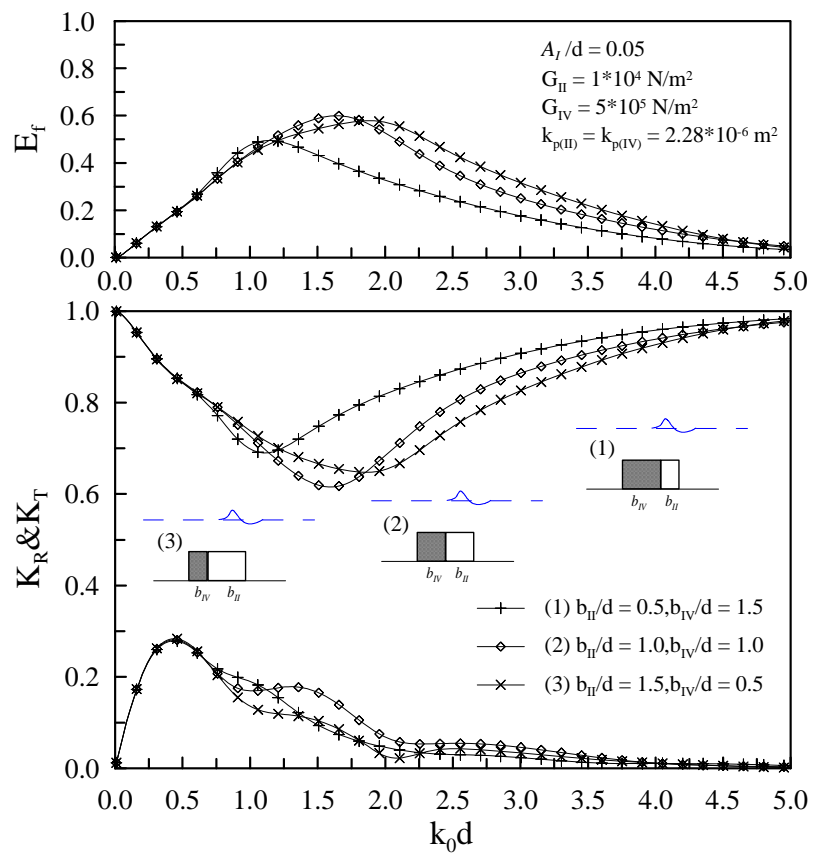

Figure 7. Effect of adjacent breakwater widths with different poro-elastic materials on $K_{R}, K_{T}, E_{f}$ $\left(G_{I I}=1 \times 10^{4} \mathrm{~N} / \mathrm{m}^{2}, G_{I V}=5 \times 10^{5} \mathrm{~N} / \mathrm{m}^{2}\right.$, and $\left.k_{p(I I)}=k_{p(I V)}=2.28 \times 10^{-6} \mathrm{~m}^{2}\right)$.
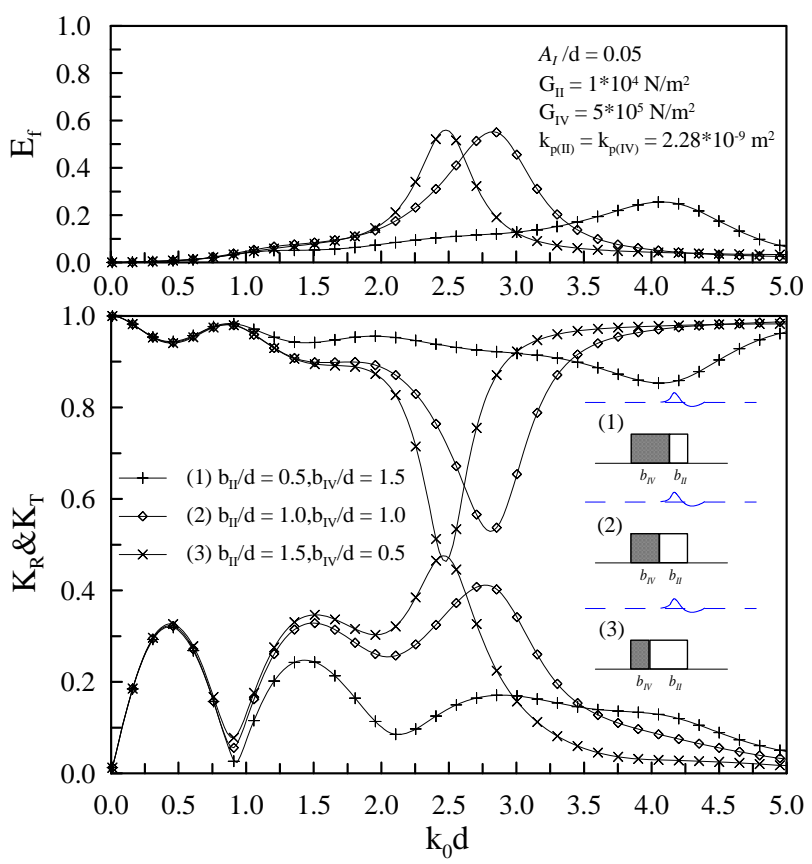

Figure 8. Effect of adjacent breakwater widths with different poro-elastic materials on $K_{R}, K_{T}, E_{f}$ $\left(G_{I I}=1 \times 10^{4} \mathrm{~N} / \mathrm{m}^{2}, G_{I V}=5 \times 10^{5} \mathrm{~N} / \mathrm{m}^{2}\right.$, and $\left.k_{p(I I)}=k_{p(I V)}=2.28 \times 10^{-9} \mathrm{~m}^{2}\right)$. 
(c) Effect of permeability with different poro-elastic materials

The permeability effect of the two closely submerged adjacent breakwaters with different poroelastic materials on wave transformation is also studied. Figures $9 \sim 11$ show the variations in $K_{R}, K_{T}$, and $E_{f}$ versus $k_{0} d$ for varying permeability in the range of $k_{p}=2.28 \times 10^{-9} \sim 2.28 \times 10^{-6} \mathrm{~m}^{2}$, where the elastic breakwater is placed upstream and various values of adjacent breakwater widths are used, including $b_{I I} / d=0.5$ and $b_{I V} / d=1.5$ in Figure $9, b_{I I} / d=1$ and $b_{I V} / d=1$ in Figure 10 , and $b_{I I} / d=1.5$ and $b_{I V} / d=0.5$ in Figure 11 .

For the case of a small width of elastic breakwater, Figure 9 shows that the wave reflection coefficient $K_{R}$ diminishes while its oscillatory interval gradually increases with the increase of permeability. For nearly impermeable breakwaters $\left(k_{p}=2.28 \times 10^{-9} \mathrm{~m}^{2}\right)$, oscillations of the transmission $K_{T}$ improve significantly and energy dissipation $E_{f}$ increases with the increase of the relative wave number $k_{0} d$. For higher values of permeability $\left(k_{p}>2.28 \times 10^{-9} \mathrm{~m}^{2}\right)$, energy dissipation decreases significantly for low values of $k_{0} d$ and the oscillation of $K_{T}$ vanishes.

For the case of a larger width of elastic breakwater, the elastic effect becomes noticeable. Figures 10 and 11 show that in the range of $k_{0} d<2.0$, the effects of the permeability on waves are similar to that shown in Figure 9. However, for permeability values less than $2.28 \times 10^{-8} \mathrm{~m}^{2}$, it is interesting to note that resonance caused by the elastic breakwater occurs for $2.5<k_{0} d<3.0$, and gradually vanishes as $k_{p}>2.28 \times 10^{-8} \mathrm{~m}^{2}$. This reduction of resonance is caused by damping effects induced by the higher seepage velocity of the interaction mechanism between the waves and submerged poro-elastic breakwaters (Lan and Lee, 2010).
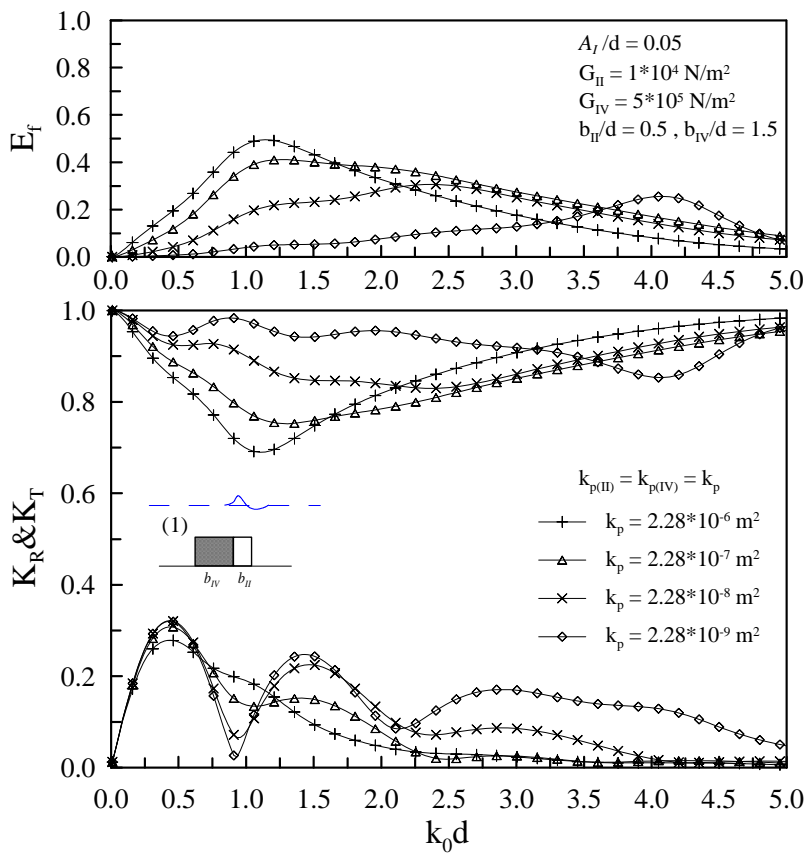

Figure 9. Effect of intrinsic permeability $k_{p}$ with different poro-elastic materials $\left(G_{I I}=1 \times 10^{4} \mathrm{~N} / \mathrm{m}^{2}\right.$, $\left.G_{I V}=5 \times 10^{5} \mathrm{~N} / \mathrm{m}^{2}\right)$ and the first combination of adjacent breakwater widths $\left(b_{I I} / d=0.5, \quad b_{I V} / d=1.5\right)$ on $K_{R}$, $K_{T}$, and $E_{f}$. 

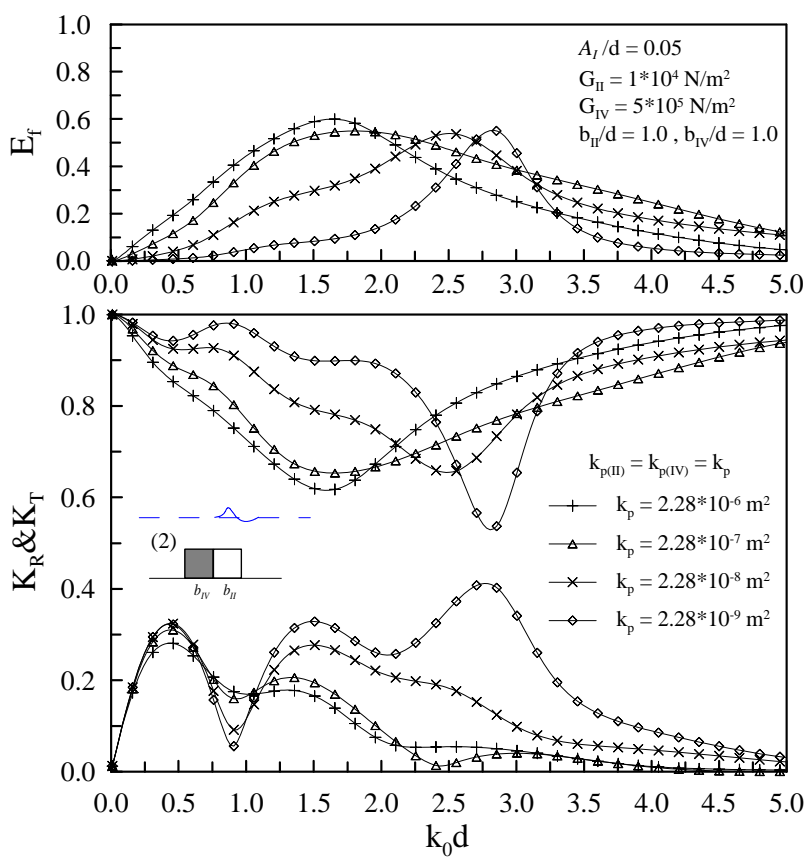

Figure 10. Effect of intrinsic permeability $k_{p}$ with different poro-elastic materials $\left(G_{I I}=1 \times 10^{4} \mathrm{~N} / \mathrm{m}^{2}\right.$, $\left.G_{I V}=5 \times 10^{5} \mathrm{~N} / \mathrm{m}^{2}\right)$ and the second combination of adjacent breakwater widths $\left(b_{I I} / d=1, b_{I V} / d=1\right)$ on $K_{R}$, $K_{T}$, and $E_{f}$.

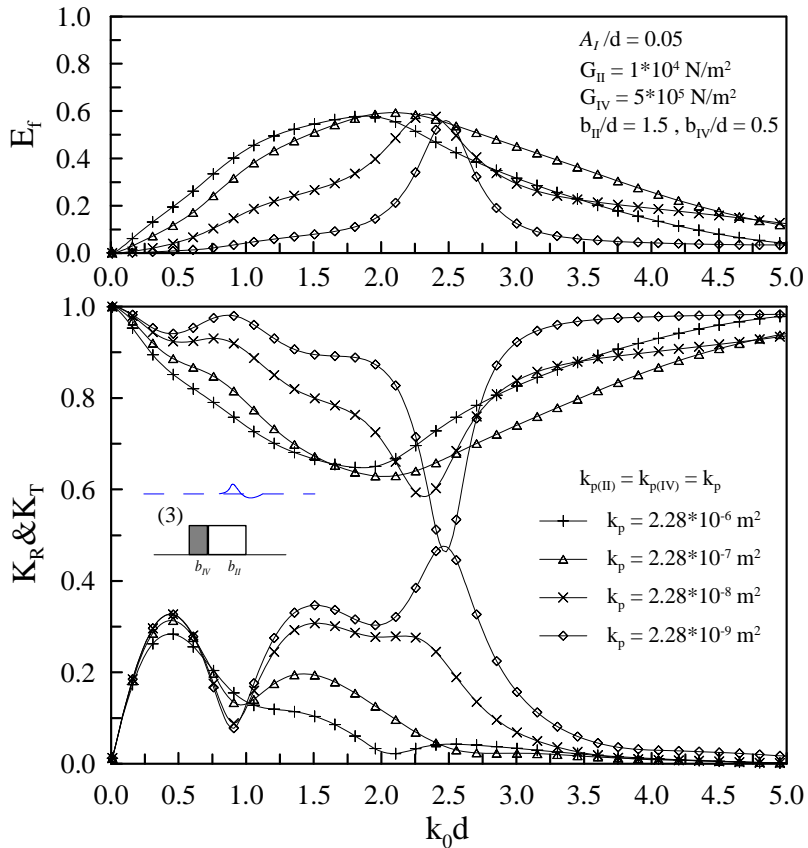

Figure 11. Effect of intrinsic permeability $k_{p}$ with different poro-elastic materials $\left(G_{I I}=1 \times 10^{4} \mathrm{~N} / \mathrm{m}^{2}\right.$, $\left.G_{I V}=5 \times 10^{5} \mathrm{~N} / \mathrm{m}^{2}\right)$ and the third combination of adjacent breakwater widths $\left(b_{I I} / d=1.5, b_{I V} / d=0.5\right)$ on $K_{R}$, $K_{T}$, and $E_{f}$. 


\section{CONCLUSION}

A theoretical formulation and an analytical solution for problem of waves propagating over two closely adjacent poro-elastic submerged breakwaters were presented in this paper. Two assumptions were made to simplify the intricate boundary conditions on the interface of adjacent poro-elastic breakwaters. To solve the linear hydrodynamic problem, an eigenfunction expansion associated with the approximation technique was employed. The results show that the analytical solution is applicable for a single submerged poro-elastic breakwater. In applying the analytical solution, wave transformation by two closely adjacent poro-elastic submerged breakwaters was intensively investigated. The parameters include the shapes of adjacent breakwaters with the same and different poro-elastic materials, and the effect of permeability with different poro-elastic materials. From the present study the following interesting findings are presented:

1. For quasi-rigid materials with the same poro-elastic properties, the two closely adjacent poroelastic submerged breakwaters can be regarded as a single submerged poro-elastic structure with homogeneous and hydraulically isotropic properties. In the softer poro-elastic medium region ( $G<10^{5} \mathrm{~N} / \mathrm{m}^{2}$ ), the structural motion mechanism on the interface of adjacent breakwaters can produce a larger wave reflection and smaller transmission than a single breakwater.

2. The largest energy dissipation occurs when the adjacent structures both have the same soft, highly permeable material and width properties.

3. In the case of elastic and rigid materials with the same permeability, wave reflection depends on the shape of two closely adjacent breakwaters, while the influence on wave transmission and energy dissipation is not significant regardless of whether the rigid breakwater is located upstream or not.

4. The resonance phenomena induced by structural vibration and wave oscillation can be found for the case of the softer and almost impermeable structure and escalates when increasing the width of the elastic breakwater. However, higher permeability produces significant wave damping and reduces the resonance on the condition of the waves and poro-elastic medium interaction.

\section{ACKNOWLEDGMENTS}

This study was financially supported by the National Science Council of Taiwan under grant numbers NSC96-2221-E-006-348-MY2 and NSC100-2221-E-006-010-MY3.

\section{REFERENCES}

Biot, M.A. 1956. Theory of propagation elastic waves in a fluid saturated porous solid. I. lowfrequency range, The Journal of The Acoustical Society of America, 28(2), 168-178.

Chen, T.W., L.H. Huang, and C.H. Song. 1997. Dynamic response of poroelastic bed to nonlinear water waves, Journal of Engineering Mechanics, 123(10), 1041-1049.

Huang, L.H., and C.H. Song. 1993. Dynamic response of poroelastic bed to water waves, Journal of Engineering Mechanics, 119, 1003-1020.

Lan, Y.J., and J.F. Lee. 2010. On waves propagating over a submerged poro-elastic structure, Ocean Engineering, 37, 705-717.

Lan, Y.J., T.W. Hsu, J.W. Lai, C.C. Chang, and C.H. Ting. 2011. Bragg scattering of waves propagating over a series of poro-elastic submerged breakwaters, Wave Motion, 48, 1-12.

Sollitt, C.K., and R.H. Cross. 1972. Wave transmission through permeable breakwaters, Proceedings of the 13th Coastal Engineering Conference, ASCE, Vancouver, 1827-1846.

Tseng, C.M., T.L. Tsai, and L.H. Huang. 2008. Effects of body force on transient poroelastic consolidation due to groundwater pumping, Environ. Geol., 54, 1507-1516.

Verruijt, A. 1969. Elastic Storage of Aquifers. Flow through Porous Media, edited by De Wiest, R.J.M., Academic, New York, 331-376.

Wang, K.H., and X. Ren. 1993. Water waves on flexible and porous breakwaters, Journal of Engineering Mechanics, 119(5), 1025-1047.

Yip, T.L., T. Sahoo, and A.T. Chwang. 2002. Trapping of surface waves by porous and flexible structures, Wave Motion, 35, 41-54. 